\title{
Frequência e fatores associados em casos confirmados e descartados e óbitos da COVID-19 em um hospital secundário
}

\section{Frequency and associated factors in confirmed and discarded cases and deaths from COVID-19 in a secondary hospital}

Maico Trevisol'1 , Bianca de Lima Piola², Claudicéia Risso Pascotto ${ }^{3}$, Leia Carolina Lucio4, Fernando Mazetto Brizola ${ }^{5}$, Guilherme Welter Wendt ${ }^{6}$, Lirane Elize Defante Ferreto ${ }^{7}$

1. ORCID: https://orcid.org/0000-0002-1389-1231. Enfermeiro. Mestrando em Ciência Aplicada à Saúde pela Universidade Estadual do Oeste do Paraná, Francisco Beltrão, Paraná, Brasil.

E-mail: maicotrevisol@hotmail.com

2. ORCID: https://orcid.org/0000-0001-8851-656X. Enfermeira. Mestranda em Ciência Aplicada à Saúde pela Universidade Estadual do Oeste do Paraná, Francisco Beltrão, Paraná, Brasil.

E-mail: biancapiola20@outlook.com

3. ORCID: https://orcid.org/0000-0003-1265-2316. Doutora em Ciências Biológicas (Biologia Celular).Docente do Programa de Pós-graduação em Ciências Aplicadas à Saúde - UNIOESTE, Francisco Beltrão, Paraná, Brasil. E-mail: claudiceiarp@hotmail.com

4. ORCID: https://orcid.org/0000-0002-8094-4188. Doutora em Ciências pela Universidade Estadual de Maringá. Docente e Docente do Programa de Pósgraduação em Ciências Aplicadas à Saúde - UNIOESTE, Francisco Beltrão, Paraná, Brasil.

E-mail: leiacarol@gmail.com

5. ORCID: https://orcid.org/0000-0002-5467-5711. Mestre em Matemática pela Universidade Federal de Santa Maria, UFSM, Brasil.

E-mail: fernando.brizola@hotmail.com

6. ORCID: https://orcid.org/0000-0002-9014-6120. Doutor em Psicologia pela Universidade de Londres (Goldsmiths College), Inglaterra.

E-mail: guilherme.wendt@unioeste.br

7. ORCID: https://orcid.org/0000-0002-0757-3659. Doutora em Saúde Coletiva. Docente do Programa de Pós-graduação em Ciências Aplicadas à Saúde UNIOESTE, Francisco Beltrão, Paraná, Brasil.

E-mail: Iferreto@hotmail.com

CONTATO: Autor correspondente: Bianca de Lima Piola | Endereço: Rua Peru, 120, Bairro Luther King, Francisco Beltrão Telefone: (46) 98403-3159 E-mail:

biancapiola20@outlook.com 


\section{RESUMO}

Diante dessa emergência de caráter pandêmico e grande impacto na Saúde Pública ocasionado pela Covid - 19, a pesquisa buscou descrever a frequência e fatores associados aos casos confirmados, descartados e óbitos da doença na abrangência da $8^{\underline{a}}$ Regional de Saúde do Estado do Paraná. Trata-se de um estudo quantitativo e transversal, que avaliou dados de 471 pacientes. A maior frequência do sexo masculino (56,7\%). Entre os casos confirmados para COVID-19, $87,5 \% \quad(n=112)$ faziam uso de suporte ventilatório não invasivo. A taxa de mortalidade calculada foi de 10,7\% (IC95\%: 7,8 - 13,4\%). A letalidade geral foi de 10,6\% (IC95\%:8,64 - 9,20) (50/471), não apresentando diferença estatisticamente significativa entre casos confirmados 10,9\% (IC95\%:8,95 - 9,25) versus 10,5\% (IC95\%:8,91 9,37 ) os descartados. Em suma, os dados corroboram a estudos prévios ao indicar uma maior ocorrência de hospitalizações em homens idosos e de elevada alteração em exames tomográficos dentre os casos confirmados da COVID-19 na instituição analisada.

DESCRITORES: Infecções por Coronavírus. Quartos de Pacientes. Hospitalização. Unidades de Terapia Intensiva.

\section{ABSTRACT}

In view of this pandemic emergency and great impact on Public Health caused by Covid - 19, the research sought to describe the frequency and factors associated with confirmed cases, discarded cases and deaths of the disease in the coverage of the 8th Regional Health Department of the State of Paraná. This is a quantitative and cross-sectional study, which evaluated data from 471 patients. The highest frequency was male (56.7\%). Among the confirmed cases for COVID-19, 87.5\% $(n=112)$ were using non-invasive ventilatory support. The mortality rate provided was $10.7 \%(95 \% \mathrm{Cl}: 7.8-13.4 \%)$. The overall lethality was $10.6 \%(95 \% \mathrm{Cl}: 8.64-9.20)(50 / 471)$, not statistically changing between confirmed cases $10.9 \%(95 \% \mathrm{Cl}: 8.95$ - 9.25$)$ versus $10.5 \%$ (95\% Cl: 8.91 - 9.37) were discarded. In short, the data corroborate previous studies by indicating a higher occurrence of hospitalizations in elderly men and high alterations in tomographic exams among the confirmed cases of COVID19 in the analyzed institution.

DESCRIPTORS: Coronavirus Infections. Patient's rooms. Hospitalization. Intensive Care Units. 


\section{INTRODUÇÃO}

1 pandemia da COVID-19 tem sido considerada um dos maiores desafios

sanitários em esfera global do século, gerando igualmente impactos diversos (econômicos, geopolíticos, etc.), embora seja ainda precoce mensurar as consequências com precisão ${ }^{1,2}$. Com efeito, a pandemia teve efeitos heterogêneos ao redor do mundo, apresentando-se, até o momento, de modo menos severo na Ásia (com exceção da Índia), na África e na Oceania do que na Europa ou na América1.

Os indicadores a nível mundial confirmaram até 13 de junho de 2021, 174. 918.667 milhões de casos com 3.782.490 de óbitos. No Brasil, a doença já acometeu 17.374.818 milhões de casos com 486.272 óbitos. O Estado do Paraná foi acometido até o momento com 1.143.636 milhões de casos com 27.976 óbitos. O coeficiente de incidência da área de abrangência $8^{\text {a }}$ Regional de Saúde é de 43.283 casos com 733 óbitos ${ }^{3}$.

O SARS-CoV-2 é o causador da COVID-19, é um vírus potencialmente contagioso. Sua transmissão ocorre através de gotículas respiratórias, contato pessoal e do contato com objetos contaminados pelo vírus, acompanhado de contato com a boca, nariz e olhos ${ }^{3,4}$. Seu período de incubação varia entre um e quatorze dias, tendo em média cinco a seis dias. Sua transmissibilidade é em média de sete dias, contudo pode ocorrer a contaminação por pacientes assintomáticos ${ }^{5}$.

Nos maiores epicentros da doença observou -se que a população acometida foi assintomática em aproximadamente (30\%), sintomas leves moderados $(55 \%)$, sintomas graves $(10 \%)$ e sintomas críticos $(5 \%)^{6}$. A disseminação da SARS-CoV-2 ocorre com maior frequência em indivíduos sintomáticos leves, moderados ou assintomáticos, que podem transmitir o vírus sem conhecimento7. Uma proporção menor de pacientes infectados tem uma apresentação grave da COVID-19, mas com desfecho elevado da mortalidade em relação aos pacientes clinicamente mais leves ${ }^{7,8}$.

Entre os principais fatores de riscos relacionados à doença estão: idade avançada (superior a 60 anos) e comorbidades como: doenças cardiovasculares, doenças pulmonares, doenças renais, obesidade, hipertensão arterial sistólica (HAS), diabetes mellitus (DM), portadores de imunossupressão, entre outras ${ }^{4,5}$. Entre seus principais sintomas encontram-se os de trato respiratório: febre, tosse, dor de garganta, fadiga. Também podem surgir sintomas como: cefaleia, calafrios, diarreia, anosmia ou hiposmia, ageusia e mialgia ${ }^{9}$. Em casos mais graves, a infecção pode levar a síndrome respiratória aguda grave (SRAG), sangramento pulmonar, lesão cardíaca, insuficiência renal, infecção secundária e choque séptico ${ }^{10,11}$. 
A taxa de letalidade está principalmente associada a pacientes com faixa etária mais elevada e indivíduos que apresentam algumas comorbidades como hipertensão arterial sistema, diabetes mellitus entre outras, também se observa que a maioria dos óbitos está associada ao sexo masculino ${ }^{5,10,12}$. Não diferente de outros lugares do mundo a $8^{\text {a }}$ Regional de Saúde do Estado do Paraná, tem sido acometida com o registro expressivo de número de casos confirmados 43.283 , e destes $15 \%$ tem sido internado em enfermarias e unidade de terapia intensiva (UTI) COVID-19.

Apesar de um elevado número de publicações, grande parte descreve a realidade de grandes centros urbanos com sistema de assistência médica hospitalar, diferenciada de regiões de pequeno porte, como a que fica localizada a $8^{\mathrm{a}}$ Regional de Saúde do Estado do Paraná. Nesta regional de saúde do Paraná incialmente foi credenciado um hospital de referência para atender pacientes suspeitos e confirmados com a doença para pacientes do Sistema Único de Saúde, e um hospital para o recebimento de casos com menor severidade, em que o paciente não necessitava de atendimento em UTI.

Sendo assim, torna-se relevante descrever as características epidemiológicas e clínicas de casos que foram internados para que esse conhecimento produzido contribua para melhor compreensão das características da doença para outras comunidades com características semelhantes a ora apresentada.

O objetivo do estudo foi de descrever frequência e fatores associados em casos confirmados, descartados e óbitos da COVID-19 em um hospital secundário de abrangência da $8^{\text {a }}$ Regional de Saúde do Estado do Paraná, em 365 dias após o registro do primeiro caso de internação da doença na instituição.

\section{MÉTODO}

Estudo seccional, com 471 pacientes hospitalizados em um hospital secundário de Francisco Beltrão - Paraná. Essa unidade hospitalar é a unidade de referência para internação clínica, responsável por uma demanda de 374.488,80 mil habitantes segundo Instituto Brasileiro de Geografia e Estatística (IBGE, 2019), que abrange os

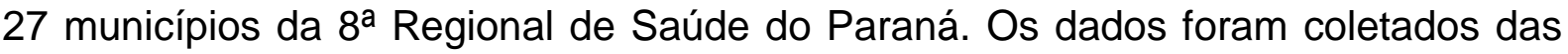
fichas de registros e prontuários médicos dos pacientes internados na instituição no período entre $1^{\circ}$ de abril de 2020 a 31 de março de 2021, devido ao uso de prontuários o uso de Termo de Consentimento Livre e Esclarecido (TCLE) foi dispensado. $O$ primeiro caso nesta unidade hospitalar de internamento com pacientes suspeito ou confirmado foi registrado em 03 de abril de 2020. 
A unidade hospitalar tem uma capacidade instalada de 86 leitos. Porém, durante o período do estudo foi isolado uma ala denominada ALA RESPIRATÓRIA contendo 20 leitos. Foram incluídos na pesquisa todos os pacientes internados com suspeita e/ou confirmados de COVID - 19 através do quadro clínico-epidemiológico ou exames de imagem como tomografias tórax e RX, notificados mediante fichas específicas de notificação. E cumprindo a determinação do ofício circular № 122/2020 - SCRACA / 8ªSS, o fluxo de regulação conforme critérios de gravidade, enquadrando a instituição em leves e moderados ${ }^{24}$.

I. Leves - Sem sinais de alerta, sem sinais de insuficiência respiratória. Tratamento domiciliar sintomático/isolamento, não realizar solicitação de internação.

II. Moderado - Sinais de alarme: saturação entre 90 e 94\% sem O2, dispneia, esforço respiratório leve, taquipnéia (FR entre 22 e 30ipm - adultos), desidratação. Tratamento no hospital do município ou pactuado, seguindo o fluxo habitual já estabelecido para internação.

A idade dos casos variou de 1 a 94 anos, com prevalência acentuada para pacientes acima de 70 anos. A distribuição etária foi heterogênea entre casos confirmados e descartados.

Neste estudo foram incluídos pacientes suspeitos e confirmados. O critério de confirmação da COVID-19 utilizado foi o laboratorial, por RT-qPCR (sigla em inglês para Reverse-TranscriptasePolymerase Chain Reactionepidemiológico).

Foram coletadas as seguintes variáveis para análise:

a) sexo (feminino; masculino);

b) idade;

c) encerramento do caso (COVID-19 confirmado ou descartado);

d) presença de comorbidades (sim ou não), para cada categoria foi agrupado em Doenças Cardiovasculares - DCV (doenças cardiológicas em geral, diabete mellitus - DM, hipertensão arterial sistólica - HAS, obesidade e outras), Doenças Pulmonares (doenças gerais do sistema pulmonar como, asma, doença pulmonar obstrutiva crônica (DPOC), atelectasia pulmonar e outras). Outras comorbidades (neoplasia, doença neurológica crônica, doença hematológica crônica, doença hepática crônica, infecção pelo vírus da imunodeficiência humana (HIV), ou seja, todas as doenças preexistentes nas mais variadas clínicas), e sem comorbidades.

e) resultado de radiografia simples ou tomografia computadorizada de tórax (com imagem em vidro fosco ou não).

f) dias de internação. 
g) evolução dos pacientes hospitalizados. Para esta variável foi avaliado a alta do paciente e/ou o óbito, como variável de desfecho.

Não houve critérios de exclusão.

Foi realizada análise descritiva com frequência, média, desvio padrão e intervalo de confiança. Foram produzidas análises bivariadas através do teste quiquadrado ou o teste exato de Fisher para as variáveis categóricas. Utilizou-se do teste binomial para comparar a taxa de mortalidade em estudo brasileiro com a taxa de mortalidade na unidade hospitalar. Considerou-se estatisticamente significativo o pvalor $<0,05$. O presente estudo compõe a pesquisa "Caracterização epidemiológica e clínica da população exposta a COVID-19: estudo transversal”, aprovada pelo Comitê de Ética em Pesquisa sob parecer número 4.034.106 de 18 de maio de 2020.

\section{RESULTADOS}

No período do estudo foram internados na ala de doenças respiratórias do hospital secundário 471 pacientes. O fluxograma do processo de definição dos pacientes está disposto na Figura 1.

Figura 1. Fluxograma dos participantes do estudo de casos confirmados e descartados da COVID-19, internados de $1^{\circ}$ de abril 2020 a 31 de março de 2021.

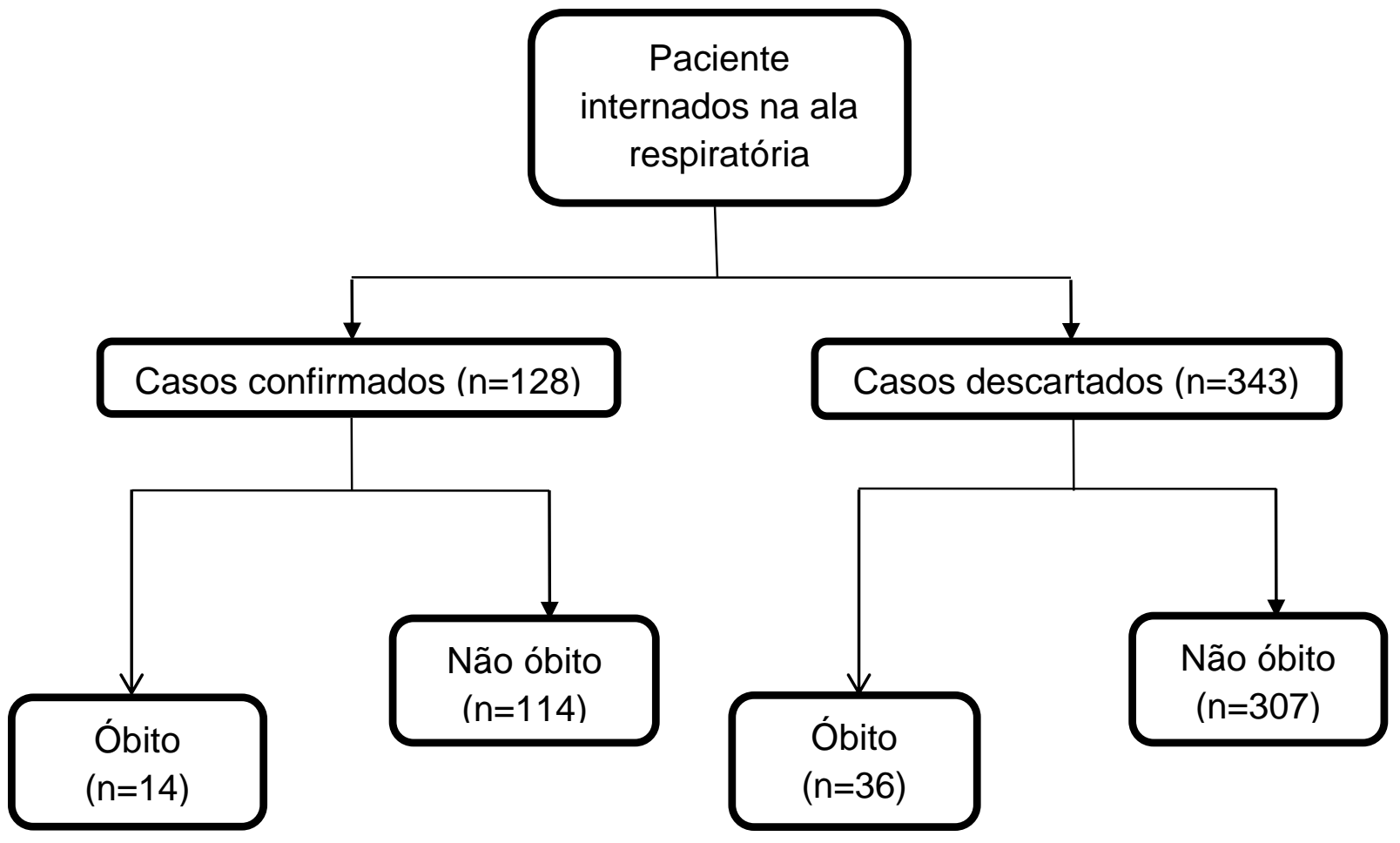

Fonte: Elaborado pelos próprios autores (2021). 
Todos os pacientes admitidos para internação na unidade hospitalar apresentavam sintomas compatíveis com a infecção da COVID-19, sendo que 86,2\% ( $\mathrm{n}=406)$ apresentavam imagem radiológica sugestiva da doença na triagem inicial, desses 27,2\% (128) positivaram para a morbidade, através do resultado reagente para RT-qPCR. Os casos suspeitos não confirmados pelo diagnóstico laboratorial foram transferidos desse setor para outras alas de internação da unidade.

Dos pacientes internados, 63,1\% ( $n=297)$ eram do município de Francisco Beltrão, sede da $8^{\text {a }}$ Regional de Saúde do Estado do Paraná, e 1,4\% de outras regionais de saúde e/ou Estado do País. A Tabela 1 apresenta o perfil dos casos notificados segundo a confirmação do diagnóstico da COVID-19. Houve predomínio do sexo masculino (61,7\%). Entre os pacientes internados do sexo masculino, 10,1\% $(\mathrm{n}=08)$ foram a óbito. A idade dos casos variou de 1 a 94 anos, com mediana de 58 anos. A distribuição etária foi heterogênea entre casos confirmados e descartados.

O uso de suporte ventilatório invasivo foi baixo, em 5,5\% (7/128) dos casos confirmados. Porém, o uso do suporte ventilatório no geral foi significativamente maior entre os confirmados. Em relação aos exames de imagem, o padrão de vidro fosco foi identificado em 124 pacientes num total de 128 dos casos confirmados, totalizando $96,6 \%$ da amostra, considerado sugestivo da infecção. A imagem de vidro fosco foi descrita também em 82,2\% (282/343) dos casos descartados.

Tabela 1. Perfil clínico epidemiológico dos casos confirmados e descartados da COVID -19 internados $(n=471)$, segundo diagnósticos da doença, internado no serviço de $1^{\circ}$ de abril de 2020 até 31 de março de $2021(n=471)$.

\begin{tabular}{lrrr}
\hline Variável & $\begin{array}{c}\text { COVID - 19 } \\
\text { Confirmado } \\
\mathbf{n}(\%)\end{array}$ & $\begin{array}{c}\text { COVID - 19 } \\
\text { Descartado } \\
\mathbf{n}(\%)\end{array}$ & \multicolumn{1}{c}{$\begin{array}{r}\text { Total } \\
\mathbf{n}(\%)\end{array}$} \\
& & & $\begin{array}{r}\text { Sexo } \\
\text { Feminino }\end{array}$ \\
Masculino & $49 / 128(38,3)$ & $155 / 343(45,2)$ & $204 / 471(43,3)$ \\
Faixa etária (anos) & $79 / 128(61,7)$ & $188 / 343(54,8)$ & $267 / 471(56,7)$ \\
$<19$ & & & \\
$20-49$ & $2 / 128(1,6)$ & $51 / 343(14,9)$ & $53 / 471(11,3)$ \\
$50-69$ & $42 / 128(32,8)$ & $57 / 343(16,6)$ & $99 / 471(21,0)$ \\
$\geq 70$ & $42 / 128(32,8)$ & $99 / 343(28,9)$ & $141 / 471(29,9)$ \\
Comorbidades & $42 / 128(32,8)$ & $136 / 434(39,7)$ & $178 / 471(37,8)$ \\
Doenças & & & \\
Cardiovasculares & $6 / 128(4,7)$ & $20 / 343(5,8)$ & $26 / 471(5,5)$ \\
Doenças Pulmonares & $3 / 128(2,3)$ & $18 / 343(5,2)$ & $21 / 471(4,5)$
\end{tabular}


Outras

Comorbidades

$6 / 128(4,7)$

$35 / 343(10,2)$

$41 / 471(8,7)$

$14 / 128(10,9)$

$33 / 343(9,6)$

$47 / 471(10,0)$

Doenças

Cardiovasculares

pulmonares

$99 / 128(21,0) \quad 237 / 343(69,1) \quad 336 / 471(71,3)$

Sem Comorbidades

0,000

Suporte ventilatório

Invasivo

$7 / 128(5,5)$

$5 / 343(1,5)$

$12 / 471(2,5)$

Não invasivo

$112 / 128(87,5) \quad 225 / 343(65,8) \quad 337 / 471(71,5)$

Sem suporte

$9 / 128(7,0) \quad 113 / 343(32,9)$

$122 / 471(25,9)$

Imagem em vidro fosco

Sim

$124 / 128(96,9) \quad 282 / 343(82,2) \quad 406 / 471(86.2)$

Não

$4 / 128(3,1)$

$61 / 343(17,8)$

$65 / 473(13,8)$

Evolução da doença

0.868

Alta $\quad 114 / 128(89,1) \quad 307 / 343(89,5) \quad 421 / 471(89,4)$

Óbito $\quad 14 / 128(10,9) \quad 36 / 343(10,5) \quad 50 / 471(10,6)$

Fonte: Elaborado pelos autores (2021).

A taxa de mortalidade geral calculada com o número de casos internados suspeitos ou com a COVID-19 e os óbitos no período foi de 10,7\% (IC95\%:7,8 13,4\%). A letalidade foi de 10,9\% (IC95\%:8,95 - 9,25) entre os casos confirmados versus 10,5\% (IC95\%:8,91 - 9,37) entre os descartados, sem diferença estatística $(p=1.000)$. A taxa de desfecho óbito para pacientes internados até 60 anos foi de $4,23 \%(p<0,01)$.

Tabela 2. Taxa de mortalidade geral dos casos confirmados e descartados da COVID19 internados $(n=421)$, segundo variáveis selecionadas de $1^{\circ}$ de abril 2020 a 31 de março de 2021

\begin{tabular}{lrrr}
\hline \multicolumn{1}{c}{ Variável } & Confirmado $\mathbf{n}(\%)$ & Descartado $\mathbf{n}(\%)$ & p-valor $^{\mathbf{a}}$ \\
$\begin{array}{l}\text { Taxa de } \\
\text { mortalidade }\end{array}$ & $14 / 114(10,9)$ & $36 / 307(10,5)$ & 1.000 \\
\hline
\end{tabular}

Fonte: Elaborado pelos autores (2021).

Em relação aos óbitos entre os casos confirmados, como descrito na Tabela 3, $57,1 \%$ (8/14) do sexo masculino evoluíram a óbito e a faixa etária superior a 70 anos em 9 casos (64,3\%), obtiveram o mesmo desfecho. Destacam-se os dias de internamento, onde 11 casos de mortalidade (78,6\%) estavam apenas em até sete dias de internamento. Observa-se que, entre os 14 casos de óbitos, 6 pacientes (42,9\%) portavam alguma comorbidade, 13 (92,9\%) apresentaram opacidade em 
vidro fosco na tomografia computadorizada de tórax e 14 (100\%) dos pacientes faziam uso algum tipo de suporte ventilatório.

Tabela 3. Evolução dos casos confirmados da COVID-19 internados $(n=128)$, segundo variáveis selecionadas de 1ํ de abril 2020 a 31 de março de 2021.

\begin{tabular}{|c|c|c|c|}
\hline Variável & $\begin{array}{l}\text { Óbito } \\
\text { n (\%) }\end{array}$ & $\begin{array}{l}\text { Não óbito } \\
\text { n (\%) }\end{array}$ & p-valor ${ }^{\mathrm{a}}$ \\
\hline Sexo & & & 0,774 \\
\hline $\begin{array}{l}\text { Feminino } \\
\text { Masculino }\end{array}$ & $\begin{array}{r}6 / 14(42,9) \\
8 / 14(57.1)\end{array}$ & $\begin{array}{r}43 / 114(37,7) \\
71 / 114(62,3)\end{array}$ & \\
\hline Faixa etária (anos) & & & 0,013 \\
\hline $\begin{array}{l}<19 \\
20-49 \\
50-69 \\
\geq 70 \\
\text { Dias de Internamento }\end{array}$ & $\begin{array}{r}0 / 14(0,0) \\
2 / 14(14,3) \\
3 / 14(21,4) \\
9 / 14(64,3)\end{array}$ & $\begin{array}{r}2 / 114(1,8) \\
40 / 114(35,1) \\
39 / 114(34,2) \\
33 / 114(28,9)\end{array}$ & 0.491 \\
\hline $\begin{array}{l}\text { Até } 7 \text { dias } \\
\text { De } 8 \text { a } 14 \text { dias } \\
\text { De } 15 \text { a } 21 \text { dias } \\
\text { Suporte ventilatório }\end{array}$ & $\begin{array}{r}11 / 14(78,6) \\
2 / 14(14,3) \\
1 / 14(7,1)\end{array}$ & $\begin{array}{r}95 / 114(83,3) \\
16 / 114(14,0) \\
3 / 114(2,6)\end{array}$ & 0,340 \\
\hline $\begin{array}{l}\text { Com suporte } \\
\text { Sem suporte } \\
\text { Imagem em vidro fosco }\end{array}$ & $\begin{array}{r}14 / 14(100,0) \\
0 / 14(0,0)\end{array}$ & $\begin{array}{r}105 / 114(92,1) \\
9 / 114(7,9)\end{array}$ & 0,374 \\
\hline $\begin{array}{l}\text { Sim } \\
\text { Não } \\
\text { Comorbidades }\end{array}$ & $\begin{array}{r}13 / 14(92,9) \\
1 / 14(7,1)\end{array}$ & $\begin{array}{r}111 / 114(97,1) \\
3 / 114(2,6)\end{array}$ & 0,054 \\
\hline $\begin{array}{l}\text { Sem comorbidades } \\
\text { Com comorbidades }\end{array}$ & $\begin{array}{r}8 / 14(57,1) \\
6 / 14(42,9)\end{array}$ & $\begin{array}{l}92 / 114(80,7) \\
22 / 114(19,3)\end{array}$ & \\
\hline
\end{tabular}

Fonte: Elaborado pelos autores (2021).

\section{DISCUSSÃO}

O objetivo da investigação foi avaliar as características e fatores associados aos casos da COVID-19. No presente estudo, entre os casos confirmados houve uma prevalência entre pacientes do sexo masculino, sendo $61,7 \%$, o que corrobora com o estudo de pesquisadores brasileiros ${ }^{14}$, onde o perfil de pessoas internadas foi $57,1 \%$ para pacientes masculinos. Estudos relatam também, que ser do sexo masculino é um fator associado à maior probabilidade do desfecho óbito em decorrência da COVID-19. A pesquisa relatou que $57,1 \%$ entre os casos de óbitos eram do sexo masculino. Esse pior desfecho tem relação com prognóstico da doença e uma maior relevância para políticas públicas, visto que, historicamente os homens apresentam 
uma resistência natural de procurar os serviços de saúde. Essa morosidade na procura dos atendimentos de saúde agrava a doença ${ }^{15}$.

A vulnerabilidade masculina em relação à COVID-19 está também relacionada aos hábitos, comportamentos, função hormonal e genética ${ }^{16}$. Citado também por pesquisadores brasileiros ${ }^{17}$, a incidência de mortalidade do gênero masculino sobressai em quase todas as populações. Nesta pesquisa acima citada, observou $58,3 \%$ na pandemia COVID -19, e resultado semelhante foi verificado na Itália $(61,9 \%)$ até o dia 29 de abril de 2020. Com isso, a diferença entre os sexos feminino e masculino, analisada por meio da razão entre as taxas específicas de mortalidade, é crescente com a idade, sendo o risco de morte dos homens cerca de duas vezes o das mulheres ${ }^{17}$.

A faixa etária predominante dos pacientes internados foi de idade $\leq 70$ anos. Em relação aos casos confirmados identificou-se a mesma proporção de pacientes internados 32,8\% nas faixas etárias de 20 a 49 anos, de 50 a 69 anos e a 70 anos respectivamente. Esses percentuais também foram relatados em outro estudo, que identificou a maior predominância da doença ocorreu entre grupos etários de 20 a 60 $\operatorname{anos}^{14}$.

Uma pesquisa realizada por brasileiros ${ }^{17}$ citou que estrutura por idade, em uma pesquisa na China mostrou que pessoas com mais de 60 anos, uma vez infectadas, experimentam maior risco de morte, isso corrobora com o trabalho onde apresentou que pacientes com idade < 70 a taxa de óbito foi 9/14 (63,3\%). Salientou também que pesquisas na Itália se observou um possível efeito da composição etária, que resulta em 95,3\% dos óbitos concentrados na população de 60 anos. No Brasil este percentual é menor $(71,4 \%)$, o que pode estar associado à estrutura etária menos envelhecida e ao perfil de comorbidades da população. Quando comparada a taxa divulgada no estudo publicado no Lancet que estimou uma taxa de óbito de pacientes internados até 60 anos em $15 \%{ }^{13}$.

Tratando-se das comorbidades, o presente estudo obteve uma frequência maior nos casos confirmados de duas doenças, sendo elas cardiovasculares e pulmonares (10,9\%) e este dado reflete que a associação de mais de uma comorbidade está diretamente relacionada ao risco maior de pior prognóstico ${ }^{10}$. $\mathrm{E}$ a pesquisa concluiu que a taxa de pacientes positivos que não apresentam comorbidades foi de $99(21,0 \%)$, e os descartados $237(69,1 \%)$. 
Outra pesquisa realizada ${ }^{12}$, quanto à presença de comorbidades, observou-se grande variação nos estudos, desde $25 \%$ dos casos em um estudo chinês a $60,5 \%$ dos casos internados na Itália, $77 \%$ dos casos hospitalizados no Reino Unido, e até cerca de $94 \%$ dos casos hospitalizados Estados Unidos. Já no Brasil, entre os casos que foram a óbito até a Semana Epidemiológica 29, o Ministério da Saúde relatou 61\% com pelo menos uma comorbidades, sendo a cardiopatia e o diabetes mellitus as mais frequentes ${ }^{18}$.

De uma forma geral, as comorbidades mais citadas nas publicações revistas foram doenças cardiovasculares, hipertensão arterial, diabetes mellitus, obesidade, doenças respiratórias, doença renal crônica e câncer ${ }^{12}$.

Quando relacionado aos casos de óbitos, $42,9 \%$ dos confirmados portavam algum tipo de comorbidade. Sabe-se que aproximadamente $80 \%$ dos pacientes com a forma grave da infecção pelo COVID-19 têm alguma comorbidade e também foram descritas complicações pulmonares e cardiovasculares decorrentes da COVID-19, como injúria miocárdica (20\% dos casos), arritmias (16\%), miocardite (10\%), além de insuficiência cardíaca e choque (até $5 \%$ dos casos), enquanto a taxa de letalidade geral por COVID-19 é de 2,3\%, nos portadores de doenças cardiovasculares ela chega a 10,5\%. Especificamente em diabéticos, esta taxa ficou $7,3 \%$ e em hipertensos, $6 \%^{19}$, dados esses dos estudos apresentado pelo autor citado.

Quando analisado o uso de suporte ventilatório, os dados sobre ventilação mecânica apresentado no estudo, somente sete pacientes que foram confirmados $(5,5 \%)$ faziam uso deste suporte, o que não corrobora a outros estudos que demonstraram que a frequência de ventilação mecânica em casos graves é sempre um percentual maior dos acometidos pela COVID-19. Porém quando relatado os óbitos entre os confirmados, $100 \%$ dos pacientes (14/14) faziam uso de algum tipo de suporte ventilatório.

O estudo realizado no Hospital Israelita Albert Einstein, em São Paulo20, descreveu características epidemiológicas e clínicas de pacientes com infecção confirmada pela SARS-CoV-2, que internou os primeiros pacientes com essa condição no Brasil. A maioria dos pacientes foi admitida na UTI por causa de insuficiência respiratória, hipoxêmica aguda que necessitou de suporte ventilatório. $A$ ventilação mecânica invasiva foi necessária em $65 \%$ dos pacientes de UTI (18,1\% do total de hospitalizações), enquanto $20 \%$ foram tratados com ventilação mecânica não invasiva. Porém estes dados da presente pesquisa refletem a realidade do local 
estudado, qual se trata de um hospital secundário, onde as internações de COVID-19 são de casos leves a moderados. Nestes casos o paciente quando faz uso de suporte ventilatório o faz na forma não invasiva. O uso desde suporte está relacionado a cateter nasal, máscara de reservatório e ventilação não invasiva.

Um dos critérios definidos na unidade hospitalar participante da pesquisa, para internação no início da pandemia quando os protocolos de atendimento ainda estavam em fase de desenvolvimento, foi à realização de tomografia computadorizada de tórax com imagens de vidro fosco, no momento atual, a realização de TC de tórax é utilizado durante o percurso da doença com forma de diagnóstico de sua evolução. A presente pesquisa relatou um percentual de $96,9 \%$ dentre os casos confirmados de pacientes com imagens de vidro fosco nas tomografias de tórax, presente também em 92,9\% dos casos de óbitos entre os confirmados, o que corrobora com o estudo realizado no Rio de Janeiro ${ }^{12}$, qual mostrou opacidade em vidro fosco superior em casos confirmados. A opacidade em vidro fosco é um achado radiológico presente desde os estágios iniciais da doença, porém também são encontradas na fase de absorção, que é caracterizada pela consolidação, no momento em que o processo inflamatório é regredido ${ }^{21}$.

Indivíduos assintomáticos podem apresentar achados pulmonares, porém com menor frequência que os pacientes sintomáticos e, geralmente, com menor acometimento e predomínio de opacidades em vidro fosco. Pacientes sintomáticos, por sua vez, apresentam mais frequentemente achados pulmonares, com predomínio de consolidações e maior extensão do acometimento parenquimatoso ${ }^{22}$.

Nos primeiros quatro dias após o início dos sintomas, predomina o padrão de opacidades em vidro fosco. Entre o $5^{\circ}$ e 0 8ํ dias, há um aumento da extensão do acometimento pulmonar, com o aparecimento de pavimentação em mosaico e consolidações $^{22}$. Entre o 9ำ e o 13ํ dias, quando costuma ocorrer o pico dos achados tomográficos, nota-se o predomínio de consolidações, e, após o 14ํำ dia a partir do início dos sintomas, começa a ocorrer a reabsorção das consolidações, o padrão de pavimentação em mosaico tende a regredir, porém podem persistir opacidades em vidro fosco 22 .

Com relação ao desfecho, a pesquisa realizada apresentou um grande percentual de alta hospitalar, sendo de $89,1 \%$ entre os confirmados, o que não corrobora com a maior parte dos estudos, visto que, demais pesquisas realizadas trazem a realidade de grandes centros de internações, principalmente em Unidades 
de Terapia Intensiva, como no estudo realizado em um hospital federal do Rio de Janeiro $^{12}$ que destacou a associação dos óbitos com a utilização de suporte ventilatório invasivo. Outro estudo também relata que o risco de morte por COVID-19 está fortemente relacionado à idade avançada e comorbidades crônicas préexistentes ${ }^{23}$.

Os casos de óbitos descartados da doença foi de 36/343 (10,5\%), consideração que a instituição é referência em clínica médica, ocasionando uma internação geral, principalmente de pessoas de mais idade, sendo que essa população possui inúmeras comorbidades, ocasionando uma taxa de óbitos considerada. Como estudo citado, onde o idoso é mais vulnerável a doenças degenerativas, como as cardiovasculares e cérebro-vasculares, as doenças oncológicas, os transtornos mentais, os estados patológicos que afetam o sistema locomotor e sentidos, havendo uma redução sistemática do grau de interação social como um dos sinais mais evidentes de velhice ${ }^{25}$.

O estudo apresenta limitações, que estão relacionadas principalmente com a utilização de dados de prontuário. Contudo, apesar das limitações este estudo forneceu fatos relevantes sobre o perfil e dados relacionados à doença, quais podem auxiliar nas estratégias de prevenção e auxiliar na prestação de serviços de saúde baseados em evidências.

\section{CONCLUSÃo}

A pesquisa relata como principal desfecho um maior percentual de alta hospitalar. Porém de acordo com a relação entre os óbitos e os casos confirmados, observa-se a predominância do sexo masculino e faixa etária superior a 70 anos, $57,1 \%$ e $64,3 \%$ respectivamente, as comorbidades seguem o mesmo curso já descrito durante um trabalho, onde 6/14 (42,9\%) pacientes confirmados que evoluíram ao óbito portavam alguma comorbidade. Entre os 14 pacientes com diagnóstico confirmado para COVID-19 que tiveram o desfecho óbito, 100\% faziam uso de suporte ventilatório e 92,9\% apresentaram opacidades em vidro fosco em tomografia de tórax. Já os casos de óbitos de pacientes descartados foram de 36 durante o desenvolvimento da pesquisa.

Neste sentido, a pesquisa corroborou ao documentar o efeito da pandemia sobre a região Sudoeste do Paraná, na sua grande maioria casos leves e moderados. 
Ademais, demostrou a eficácia do sistema de saúde, sua importância no cotidiano e, principalmente, em momentos pandêmicos.

\section{REFERÊNCIAS}

1. Bramajo $O$, Infantino $M$, Unda $R$, Cardona-Maya WD, Richly $P$. The role of population structure when measuring COVID-19 impact across countries [Internet]. Epidemiology; 2020 dez [citado 5 de julho de 2021]. Disponível em: http://medrxiv.org/lookup/doi/10.1101/2020.11.30.20239947

2. Werneck GL, Carvalho MS. A pandemia de COVID-19 no Brasil: crônica de uma crise sanitária anunciada [Internet]. Cad Saúde Pública. 2020 [citado em 12 de julho de 2021]. doi: https://doi.org/10.1590/0102-311X00068820

3. SESA - Secretaria da Saúde. Boletim - Informe Epidemiológico Coronavírus (COVID-19) [Internet]. Paraná; 2021 [citado em 12 de junho de 2021]. Disponível em: https://www.saude.pr.gov.br/sites/default/arquivos restritos/files/documento/2021 -06/informe epidemiologico 1306 2021.pdf

4. Campos MR, Schramm JMA, Emmerick ICM, Rodrigues JM, Avelar FG de, Pimentel TG. Carga de doença da COVID-19 e de suas complicações agudas e crônicas: reflexões sobre a mensuração (DALY) e perspectivas no Sistema Único de Saúde [Internet]. Cad Saúde Pública. 2020 [citado em 12 de julho de 2021]. doi: https://doi.org/10.1590/0102-311X00148920

5. Marins AMF, Domingos AM, Duarte SCM, Gaspar RB, Abreu SF, Carvalho LQ. A saúde da pessoa idosa no contexto da pandemia pelo Coronavírus: considerações para a enfermagem. Rev Enferm Cent-Oeste Min [Internet]. 14 de outubro de 2020 [citado 15 de abril de 2021];10(0). doi: https://doi.org/10.19175/recom.v10i0.3789

6. Guan W, Ni Z, Hu Y, Liang W, Ou C, He J, et al. Clinical Characteristics of Coronavirus Disease 2019 in China [Internet]. N Engl J Med. 2020 [citado em 12 de julho de 2021]. doi: https://doi.org/10.1056/NEJMoa2002032

7. Lai C-C, Liu YH, Wang C-Y, Wang Y-H, Hsueh S-C, Yen M-Y, et al. Asymptomatic carrier state, acute respiratory disease, and pneumonia due to severe acute respiratory syndrome coronavirus 2 (SARS-CoV-2): Facts and myths [Internet]. J Microbiol Immunol Infect. junho de 2020 [citado em 12 de julho de 2021]. doi: https://doi.org/10.1016/j.jmii.2020.02.012

8. WHO Coronavirus (COVID-19) Dashboard | WHO Coronavirus (COVID-19) Dashboard With Vaccination Data [Internet]. [citado 22 de abril de 2021]. Disponível em: https://COVID19.who.int/table

09. Fernandes F, Ramires FJA, Fernandes FD, Simões MV, Mesquita ET, Mady C. Afecções Pericárdicas em Pacientes com COVID-19: Uma Possível Causa de Deterioração Hemodinâmica [Internet]. Arq Bras Cardiol. 18 de setembro de 2020 [citado em 12 de julho de 2021]. doi: https://doi.org/10.36660/abc.20200474

10. Xavier AR, Silva JS, Almeida JPCL, Conceição JFF, Lacerda GS, Kanaan S. COVID-19: clinical and laboratory manifestations in novel coronavirus infection. $J$ 
Bras Patol E Med Lab [Internet]. 2020 [citado 15 de abril de 2021]; doi: https://doi.org/10.5935/1676-2444.20200049

11. Strabelli TMV, Uip DE. COVID-19 e o Coração: C. Arq Bras Cardiol [Internet]. 2020 [citado 15 de abril de 2021]; doi: https://doi.org/10.36660/abc.20200209

12. Escosteguy CC, Eleuterio TA, Pereira AGL. COVID-19: a cross-sectional study of suspected cases admitted to a federal hospital in Rio de Janeiro, Brazil, and factors associated with hospital death [Internet]. Epidemiol. Serv. Saude. 2021 [citado em 12 de julho de 2021]. doi: https://doi.org/10.1590/S1679-49742021000100023

13. Ranzani OT, Bastos LSL, Gelli JGM, Marchesi JF, Baião F, Hamacher S, et al. Characterisation of the first 250000 hospital admissions for COVID-19 in Brazil: a retrospective analysis of nationwide data [Internet]. Lancet Respir Med. $1^{\circ}$ de abril de 2021 [citado em 12 de julho de 2021]. doi: https://doi.org/10.1016/S22132600(20)30560-9

14. Araújo Filho ACA, Arrais KR, Silva MSG da, Arrais KR, Monteiro AKC, Silva AP. Análise de casos confirmados e óbitos pelo novo Coronavírus no Piauí / Analysis of confirmed cases and deaths by the new Coronavirus in Piauí. J Nurs Health [Internet]. 24 de novembro de 2020 [citado 15 de abril de 2021];10(4). Disponível em: https://periodicos.ufpel.edu.br/ojs2/index.php/enfermagem/article/view/19940

15. Galvão MHR, Roncalli AG. Fatores associados a maior risco de ocorrência de óbito por COVID-19: análise de sobrevivência com base em casos confirmados [Internet]. Rev Bras Epidemiol. 2020 [citado em 12 de julho de 2021]. doi: https://doi.org/10.1590/1980-549720200106

16. Sousa AR, Santana TS, Carvalho ESS, Mendes IAC, Santos MB, Reis JL, et al. Vulnerabilities perceived by men in the context of the COVID-19 pandemic [Internet]. Rev Rene. 4 de janeiro de 2021 [citado em 12 de julho de 2021]. doi: https://doi.org/10.15253/2175-6783.20212260296

17. Souza LG, Randow R, Siviero PCL. Reflexões em tempos de COVID-19: diferenciais por sexo e idade [Internet]. Comun Em Ciênc Saúde. 20 de maio de 2020 [citado em 12 de julho de 2021]. Disponível em: http://www.escs.edu.br/revistaccs/index.php/comunicacaoemcienciasdasaude/art icle/view/672

18. SESA - Secretaria da Saúde. Boletim - Informe Epidemiológico Coronavírus (COVID-19). Paraná; 2020 [citado em 12 de junho de 2021]. Disponível em: https://www.saude.pr.gov.br/Pagina/Coronavirus-COVID-19

19. Costa IBSS, Bittar CS, Rizk SI, Araújo Filho AE, Santos KAQ, Machado TIV, et al. O Coração e a COVID-19: O que o Cardiologista Precisa Saber [Internet]. Arq Bras Cardiol. 22 de maio de 2020 [citado em 12 de julho de 2021]. doi: https://doi.org/10.36660/abc.20200279

20. Teich VD, Klajner S, Almeida FAS de, Dantas ACB, Laselva CR, Torritesi MG, et al. Características epidemiológicas e clínicas dos pacientes com COVID-19 no Brasil [Internet]. Einstein Sao Paulo. 12 de agosto de 2020 [citado em 12 de julho de 2021]. doi: http://dx.doi.org/10.31744/einstein journal/2020A06022

21. Mogami R, Lopes AJ, Araújo Filho RC, Almeida FCS, Monteiro A. Chest computed tomography in COVID-19 pneumonia: a retrospective study of 155 patients at a 
university hospital in Rio de Janeiro [Internet], Brazil. 2021 [citado em 12 de julho de 2021]. doi: https://doi.org/10.1590/0100-3984.2020.0133

22. Chate RC, Passos RBD, Shoji H, Szarf G. Apresentação tomográfica da infecção pulmonar na COVID-19: experiência brasileira inicial [Internet]. J Bras Pneumol [citado em 12 de julho de 2021]. doi: https://dx.doi.org/10.36416/1806$\underline{3756 / \mathrm{e} 20200121}$

23. Elezkurtaj S, Greuel S, Ihlow J, Michaelis EG, Bischoff P, Kunze CA, et al. Causes of death and comorbidities in hospitalized patients with COVID-19 [Internet]. Sci Rep. dezembro de 2021 [citado em 12 de julho de 2021]. doi: https://doi.org/10.1038/s41598-021-82862-5

24. SESA. Secretária de Saúde. Ofício circular no 122/2020/SCRACA/8ªRS. Francisco Beltrão,19 mai. 2020 (Ofício não publicado).

25. Zaslavsky C, Gus I. Idoso. Doença Cardíaca e Comorbidades [Internet] Arq Bras Cardiol. 2002 [citado em 15 de novembro de 2021]. doi: https://doi.org/10.1590/S0066-782X2002001500011 